\title{
IMPLEMENTATION OF ISO 9001:2000 QUALITY MANAGEMENT SYSTEM AS APPLIED TO GAMMA SCANNING TECHNIQUE FOR INSPECTING FRACTIONNING COLUMNS AND RELATED UNIT PROCESS OPERATIONS
}

\author{
Carlos SEBASTIÁN CALVO \\ Universidad Ricardo Palma \\ csebastian@urp.edu.pe
}

\begin{abstract}
Product manufacturers and service providers have to follow certain procedures and protocols to guarantee the quality products and services to satisfy consumer requirements. Standards contribute by making development, manufacturing and supply of products and services more efficient, safer and competitive.

The quality assessment and quality control system in radioisotope applications consists of enhanced quality awareness, better presentation of documentation, establishment of standard operating manuals, and preparation of procedures for validation of methods, surveillance of method performance and formulation of protocols for experimental design and execution, regular qualification of personnel, client liaison and safety.

The gamma logging to be performed consists of moving, simultaneously, a radioactive source and a detector along the study column. Both tend to be located (although it is not the single possibility) as diametrically opposed, leaving the column interposed between the two. Liquid, gas, foam, dishes, packaging and any other component inside of the column will attenuate the beam of radiation in varying degrees. Consequently, detector response will be plotted as a function of height to obtain a vertical density profile. An abnormal density profile and the causes of malfunctioning can be identified through the analysis of such a profile.

Production of quality column scanning services depend on various parameters that include the use of competent manpower, reliable equipment, proper procedures and working instructions and conducive environment as well as good working practice. Thus, to achieve the quality in column scanning service, requires systematic and planned activities that take into consideration all factors.

Personnel in the column scanning team are trained on various disciplines in gamma-ray scanning activities. Basic training is carried out in a systematic manner to enable worker to adopt the techniques required to obtain a quality column scanning services, as appropriate to the duties. Supervisor training is also essential to further integrate column scanning service procedures and work practices into the day-to-day staff activities.
\end{abstract}




\section{KEYWORDS}

Gamma scanning, Density profile, Malfunctioning, ISO 9001:2000, Certification, National or International body, Quality Assurance/Quality Management, Accreditation

Recibido: $5 / 5 / 2017$

Aprobado: $30 / 11 / 2017$

\section{Introduction}

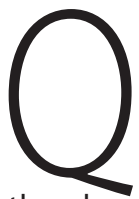

uality control and assurance of products and services are needs of the day. The product manufacturers and service providers have to follow certain prescribed procedures and protocols to guarantee quality products and services to consumers and clients. To meet this requirement they need authentic certification so that they have to follow acceptable national/international standards. Standards contribute to making the development, manufacturing and supply of products and services more efficient, safer and competitive. Standards also make trade between countries easier.

The quality assessment and quality control (QA/QC) system in radioisotope applications consists of enhanced quality awareness, better presentation of documentation, establishment of standard operating manuals, preparation of procedures for validation of methods, surveillance of method performance, formulation of protocols for experimental design and execution, regular qualification of personnel, client liaison and safety. The QA/ QC system once established promotes radioisotope laboratories to maintain a self-sufficient quality system by which they might be able to obtain national or international accreditation. $\mathrm{QA} / \mathrm{QC}$ is a continuous process, not a goal to be achieved.

Radioisotope techniques are highly useful tools to investigate variety of problems in petrochemical industry. Major techniques and applications are in routine service to industry. Although the technology has been refined in terms of equipment and technical expertise over the years, yet the techniques and laboratories are not certified by a standard national/international body.

Gamma-ray scanning, often referred to as "column scanning", is a convenient, cost effective, fast, efficient and non-intrusive technique to examine inner details of a process column or a reaction vessel, while it is in operation. Column scanning allows an engineer in petroleum refineries, gas processing installations and chemical plants, to study tray or packing hydraulics inside a distillation column at any set of on-line condition. It provides essential data to optimize the performance of columns, extend column run times, track the performance-deteriorating effects of fouling and solids deposition, and identify maintenance requirements well in advance of scheduled turnarounds. This on-line information can reduce repair downtimes significantly. 


\section{Technology aspects}

When some material is found between a gamma radiation source and a detector, the original radiation intensity is reduced because of the different interactions that take place between the gamma photons and atoms of the material. By keeping constant other factors, the degree of attenuation suffered by the incident radiation becomes a function of the density of the filed material.

The gamma logging to be performed consists of moving, simultaneously, a radioactive source and a detector along the study column. Both tend to be located (although it is not the single possibility) as diametrically opposed, leaving the column interposed between the two. Liquid, gas, foam, dishes, packaging and any other component inside of the column will attenuate the beam of radiation in varying degrees. Consequently, detector response will be plotted as a function of height to obtain a vertical density profile. The existence of problems affecting the proper functioning of a column, will result in several alterations in the density profile and the causes of malfunctioning can be identified through the analysis of a gamma profile.

It is possible to determine the following anomalies: tray drop (one of the dishes detaches totally or partially), flooding (a blockage avoids the normal displacement of fluids into the lower trays), dragging (pressure difference between a tray and the immediate upper tray is very high, so that the entering fluid is dragged upwards mixing again liquid and gases), rain (displacement of the fluid by gas pipelines, probably damaged, from the active part of a tray to the lower one), foam (the appearance of gas bubbles on the surface of the liquid, makes difficult the displacement of gas upward, reducing the quality of separation from the tray), among other malfunctioning troubles. (See figure 1).
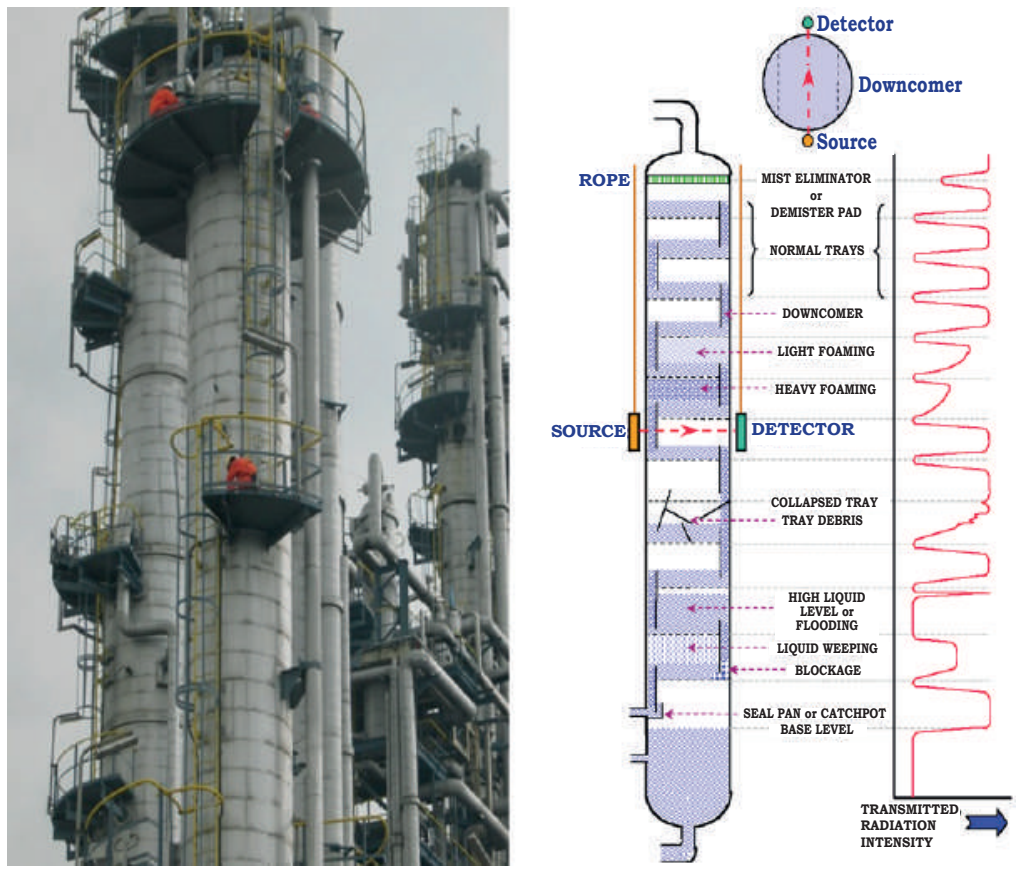

Fig.1 Typical column scanning (gamma-ray scanning) services 
The basic device for gamma scanning consists of a semi-automatic moving system, allowing displacement of both, radioactive source and radiation detector, simultaneously and longitudinally along the column. The equipment is completed with a simple data acquisition system coupled to a "lap-top" type computer or similar. Field tasks will begin with the installation of the motion system consisting of a metal structure with two reels driven by two handles that allow to spool a pair of steel cables that hold the detector probe and the source holder, respectively. Two pulley blocks, through which passed the source and the detector steel cables, are placed at the level of bottom column, while the structure with the reel is located at a suitable distance to reduce the radiation dose to negligible values.

Figure 2 shows a picture of the motion system while in figure 3 an outline of the assembly is showed. It must be taken into account that the height of a distillation column can have several tens of meters.

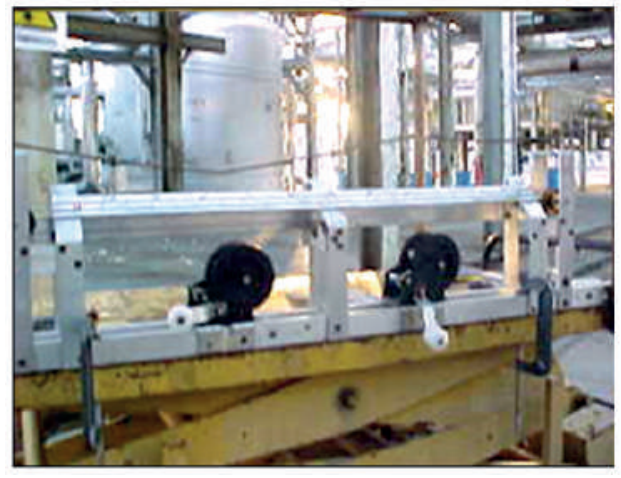

Fig.2 Moving system

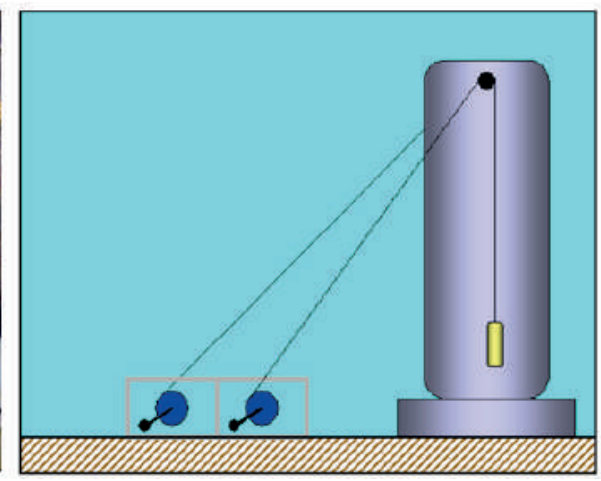

Fig.3 Installing of the moving system

The work area, around the tower is closed through a fence to prevent the access of non-operation staff and, after that, proceed to the transfer of radioactive gamma source, such as a cobalt-60 source, from its original container for transport to its holding source. This consists of a stainless steel tube that houses two cylinders of lead, and, inside them, a third acrylic cylinder with a central hole where the radioactive source is located. Figure 4 shows the holding source. 

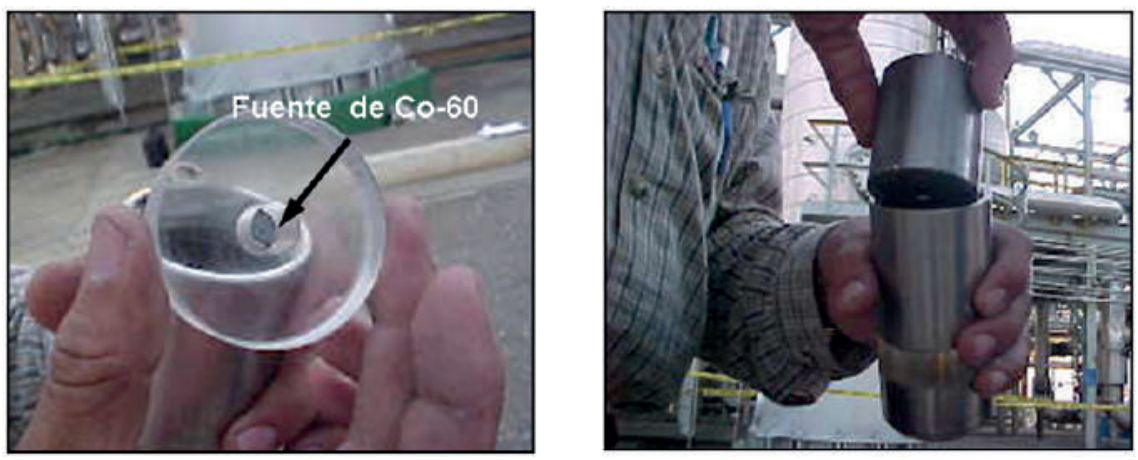

Fig.4 Source holder

The next step will consist of a holder source setting at the end of one of the steel cables, taking into account that the other will be set the detector probe. Then, a reference measurement in a suitable area next to the bottom of the column is performed. Finally, a manually moving profile is performed by activating the respective cranks. A movement must be made by steps at fixed intervals (usually $5 \mathrm{~cm}$ or $10 \mathrm{~cm}$ ), depending on the type and dimensions of each column.

Completed the operation, the system is disassembled. The readings remain stored in the computer's memory for later analysis.

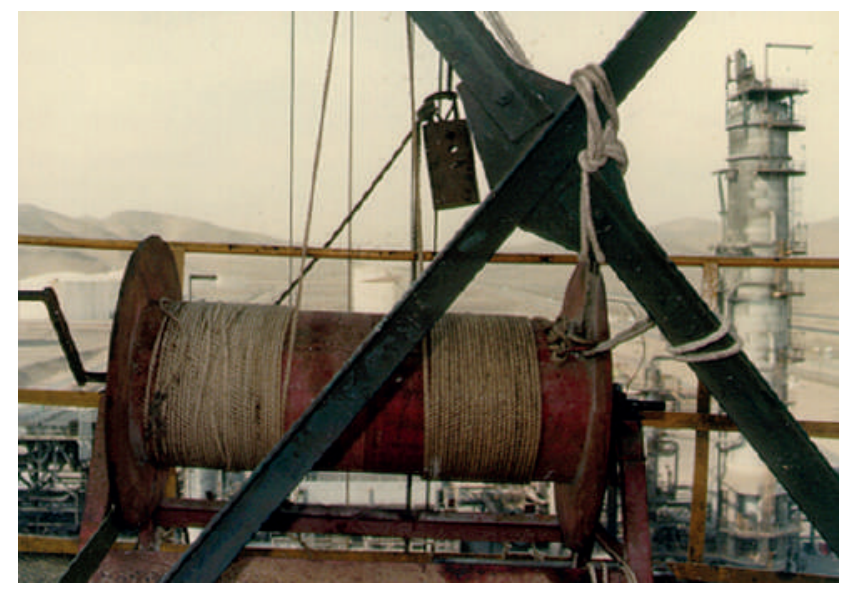

Fig.5 Gamma scanning of a Peruvian oil refinery column. Observe the mechanical system used for the simultaneously displacement the source and the detector (reel wrapping wires that suspend both, the source and the detector, powered by a single shaft). 


\section{Scanning desing criteria}

\subsection{Fundamentals}

The following procedures are performed to mount the gamma scanning system:

(i) Obtain detailed mechanical schemes of the column.

(ii) Request for assistance of a process engineer for details of the process.

(iii) Decide the orientation of the scan and the number of scanning lines.

(iv) Check the source and detector alignment every $50 \mathrm{~cm}$ of the scanning.

(v) Check any unusual decrease in the intensity of the radiation due to external obstruction in the column.

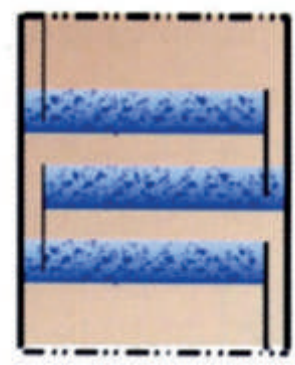

Simple tray pass

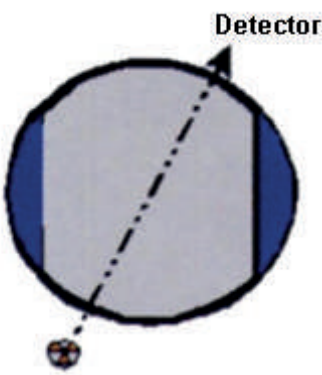

Radioactive source

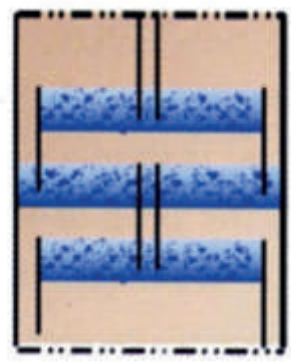

Double tray pass

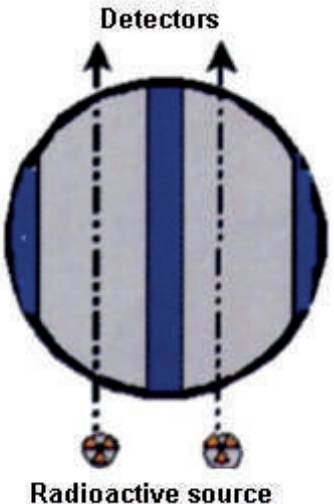

Radioactive source

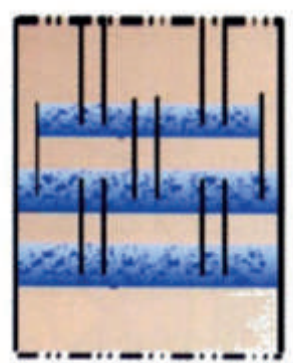

Multi tray pass

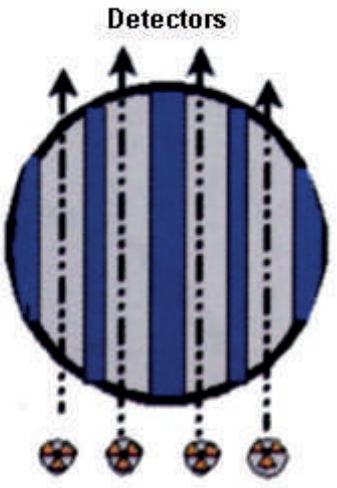

Radioactive source

Fig.6 Different column trays, scan directions and scanning lines showing typical transmitted radiation 


\subsection{Case study: Depropanizer column from a natural gas liquid fractioning plant}

\begin{tabular}{|c|c|c|c|c|}
\hline \multirow[t]{3}{*}{$\begin{array}{l}\text { Characteristics } \\
\text { of the column }\end{array}$} & $\begin{array}{c}\text { Type of } \\
\text { the part } \\
\text { to be } \\
\text { inspected }\end{array}$ & $\begin{array}{c}\text { Highest } \\
\text { external } \\
\text { diameter, } \\
\text { with } \\
\text { coating } \\
(\mathrm{mm}) \\
\end{array}$ & $\begin{array}{l}\text { Max. wall thickness } \\
(\mathrm{mm})\end{array}$ & Nature of components \\
\hline & \begin{tabular}{|l}
$\begin{array}{l}\text { Complex } \\
\text { column }\end{array}$ \\
\end{tabular} & 3250 & 32 & Liquid and vapor phases \\
\hline & \begin{tabular}{|l|}
3 types of \\
trays
\end{tabular} & - & - & - \\
\hline \multirow{3}{*}{ Source } & Nature & $\begin{array}{l}\text { Activity } \\
\text { (mCi) }\end{array}$ & Lead shielding (mm) & Remarks \\
\hline & Co-60 & 50 & $5 \mathrm{~cm}$ & - \\
\hline & Gamma & 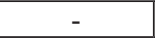 & - & - \\
\hline \multirow[b]{2}{*}{$\begin{array}{l}\text { Configuration of } \\
\text { the scan lines }\end{array}$} & \multicolumn{3}{|c|}{ Orientation of scan } & Observations \\
\hline & OF & & TION 2, STRE] & $\begin{array}{l}\text { Option 1.- Measure 1,64 m. } \\
\text { starting from the projection of } \\
\text { the center of upper downcom- } \\
\text { er, to both sides of the circum- } \\
\text { ference. Then install source } \\
\text { and detector. } \\
\text { Option 2.- Make the measure- } \\
\text { ments in the same way, from } \\
\text { the opposite side. }\end{array}$ \\
\hline \multirow{2}{*}{ 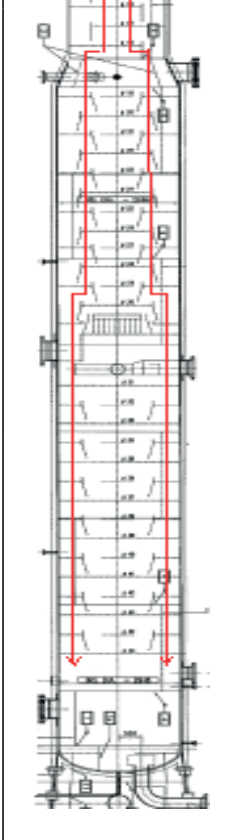 } & $\begin{array}{c}\text { OPTION } \\
\text { D }\end{array}$ & STRETCH 2 & OPTION 2, STRET & $\begin{array}{l}\text { Option 1.- Change direction } \\
\text { before reaching the height of } \\
\text { the plate } 19 \text {, when diameter } \\
\text { of the column I change. Con- } \\
\text { tinue and make a new change } \\
\text { before the plate } 32 \text {, until the } \\
\text { bottom of the column. } \\
\text { Option 2.- Make the measure- } \\
\text { ments in the same way, from } \\
\text { the opposite side. }\end{array}$ \\
\hline & $\begin{array}{l}\text { OPTION 1, } \\
\text { D }\end{array}$ & STRETCH 3 & $\begin{array}{c}\text { OPTION 2, STRETCH } 3 \\
\mathrm{D}\end{array}$ & $\begin{array}{l}\text { Option 1.- Continue the scan } \\
\text { and make a new change of } \\
\text { direction before reaching the } \\
\text { plate } 32 \text {. Continue until reach- } \\
\text { ing the bottom of the column. } \\
\text { Option 2.- Make the measure- } \\
\text { ments in the same way, from } \\
\text { the opposite side. }\end{array}$ \\
\hline
\end{tabular}




\subsection{Scanning by coordinates as applied to packed columns}

Some columns have fixed or mixed bed. The scanning by coordinates will be the recommended for fixed bed or fill columns. A typical orientation of coordinates scan lines is shown in figure 7. At least two scanning will be necessary to analyze fixed bed columns. Columns of large diameters will be scanned in a different way, at least in four sweeps. The scanning by coordinates will be used to investigate conditions related to processes such as:

(i) Obstructions or flooding.

(ii) Input overflow or liquid transport.

(iii) Unequal distribution of fluid through the fixed beds.

The scanning by coordinates can also be used to investigate design mechanical problems such as the collapsed fixed beds or non-uniform distribution of packaging material.

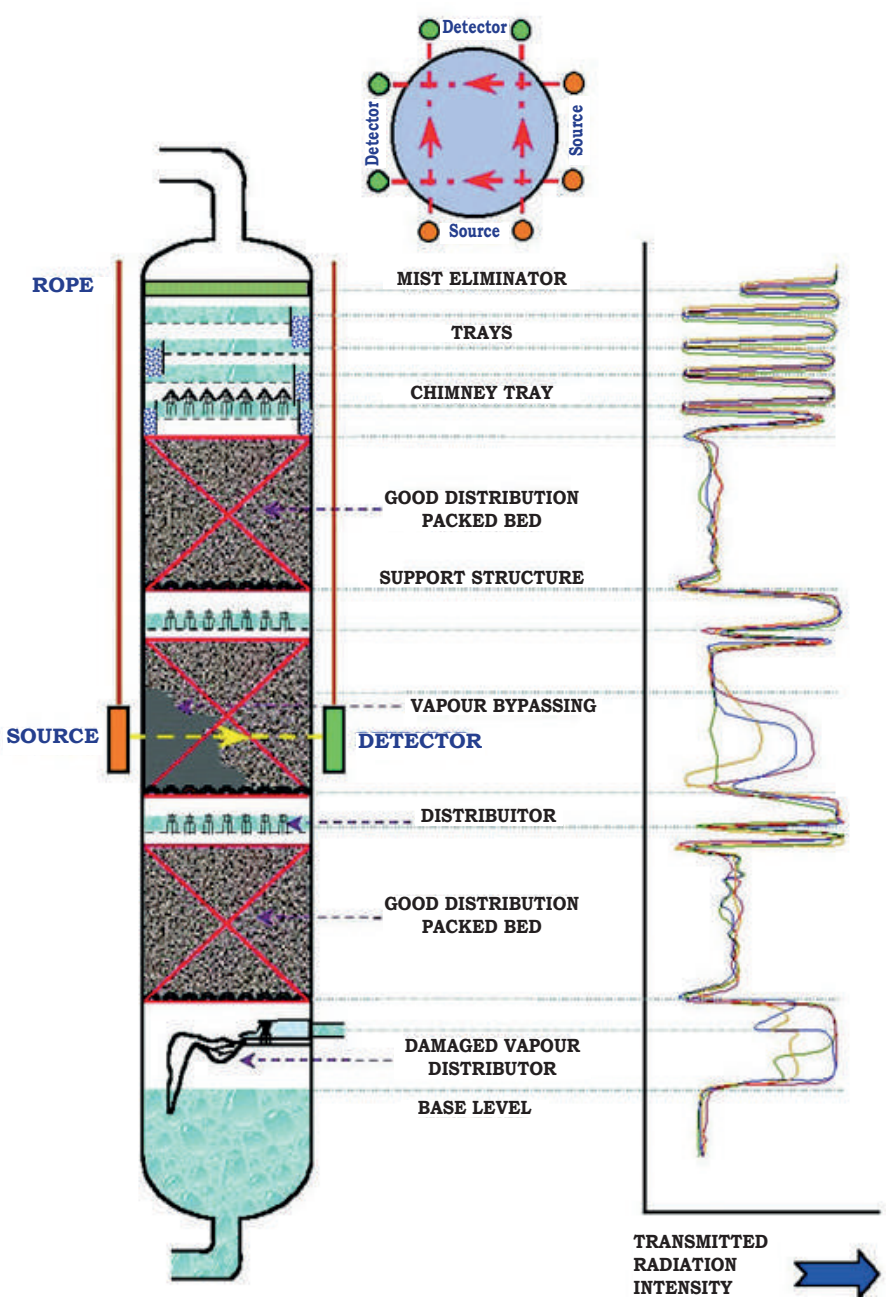

Fig.7 Gamma scanning lines, applicable to packed bed columns

Scanning by coordinates packed bed columns will be useful also to investigate the proper installation of demister distributers as well as the correct distribution of the liquid feeding. An 
irregular distributor can affect the performance of all the packed bed and the column. Liquid distributors must distribute fluid uniformly over the bed, resist clogging and dirtiness, and also to provide clearance for gas flow. An incorrect level of water at the installed distributor, tilted in this case, can result in preferably flows in only one side of the column.

\subsection{Dose Calculations}

For dose calculation two situations are considered: the transfer of the source from its container to the source holder and the transfer of this to the column under study, and its later fixation in the wire rope's movement system. For the first operation, the dose on hands will be evaluated and, for the second, in hands and whole body.

For the transfer of the source from its original container to the source holder, these elements will be placed behind a wall of lead and the operation shall be carried out by a telemeter of about $40 \mathrm{~cm}$ length. It is estimated that 30 seconds will be enough time to complete the task.

Later on, the source holder will be closed by a lead cylinder and a lid of stainless steel (Figure 4) and moved to the bottom of column where it is tight to the moving systemt. Another 30 seconds will sufficient to complete this operation. The source will be approximately $15 \mathrm{~cm}$ from the hand and $50 \mathrm{~cm}$ from the body.

By considering a source of $1.85 \mathrm{GBq}$ of ${ }^{60} \mathrm{C}_{0}$, the results are listed below.

\begin{tabular}{|l|c|c|c|c|c|}
\hline \multicolumn{6}{|c|}{ OPERATIONS WITH THE SOURCE OF ${ }^{60}$ Co , 1,85 GBq } \\
\hline \multirow{2}{*}{ Operation } & Distance & Dose rate & Time & Dose \\
\cline { 3 - 6 } & $\mathbf{c m}$ & mSv / hour & Seconds & $\boldsymbol{\mu S v}$ \\
\hline \multicolumn{2}{|c|}{ Transfer (hands) } & 40 & 4,09 & 30 & 34,1 \\
\hline \multirow{2}{*}{ Transport } & hands & 15 & 29,1 & 30 & 242,5 \\
\cline { 2 - 6 } & body & 50 & 2,62 & 30 & 21,8 \\
\hline \multirow{2}{*}{ Total dose } & hands & & & & $\mathbf{2 7 6 , 6}$ \\
\cline { 2 - 6 } & body & & & & $\mathbf{2 1 , 8}$ \\
\hline
\end{tabular}

\section{ISO 9001:2000 Quality Management System For Column Scanning Services}

Column scanning activities were introduced some decades ago. Since then, it is largely used in routine services in many Latin American countries such as Argentina, Brazil, Ecuador, Mexico, Peru and Venezuela. Qualified technical staffs have performed hundreds of scans on various types of process columns in petroleum refineries, gas processing installations and chemical plants. Scans are frequently carried out on spot-check basis to investigate column malfunctions, and many are scanned on a regular basis to provide early warning of deterioration in their performance.

Some service companies or organizations are no longer restricting their activities within the country. Column scanning services could be further extended to other countries within the region, in particular to countries in Central America. In this case, quality column scanning services and laboratories will certainly enhance the image and would provide a strong point of acceptance by clients abroad. 


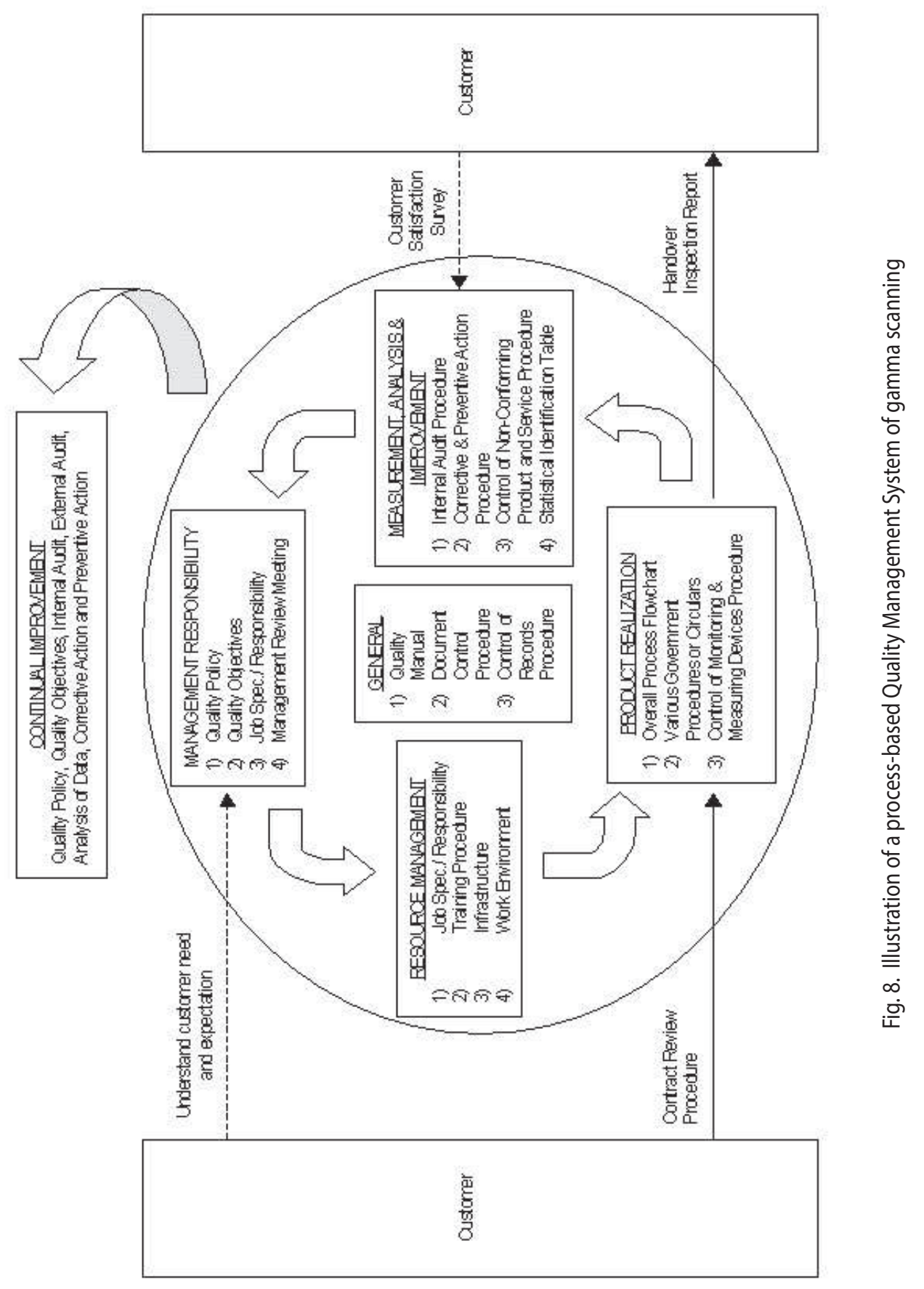


Production of quality column scanning services depend on various parameters that include the use of competent manpower, reliable equipment, proper procedures and working instructions and conducive environment as well as good working practice. Thus, to achieve quality in column scanning service, it requires systematic and planned activities that take into consideration all factors. In other words, quality service requires an effective quality management system of all activities that contribute to the production of the scanning data or results.

The model recognizes the fact that customers and other interested parties play a significant role during the process of defining input requirements. Process management is then implemented for all the processes required to realize the required product and/ or service and the process outputs are verified. Customer satisfaction assessment and satisfaction evaluation of other relevant interested parties, are used as a guide to evaluate and validate whether customer requirements have been achieved.

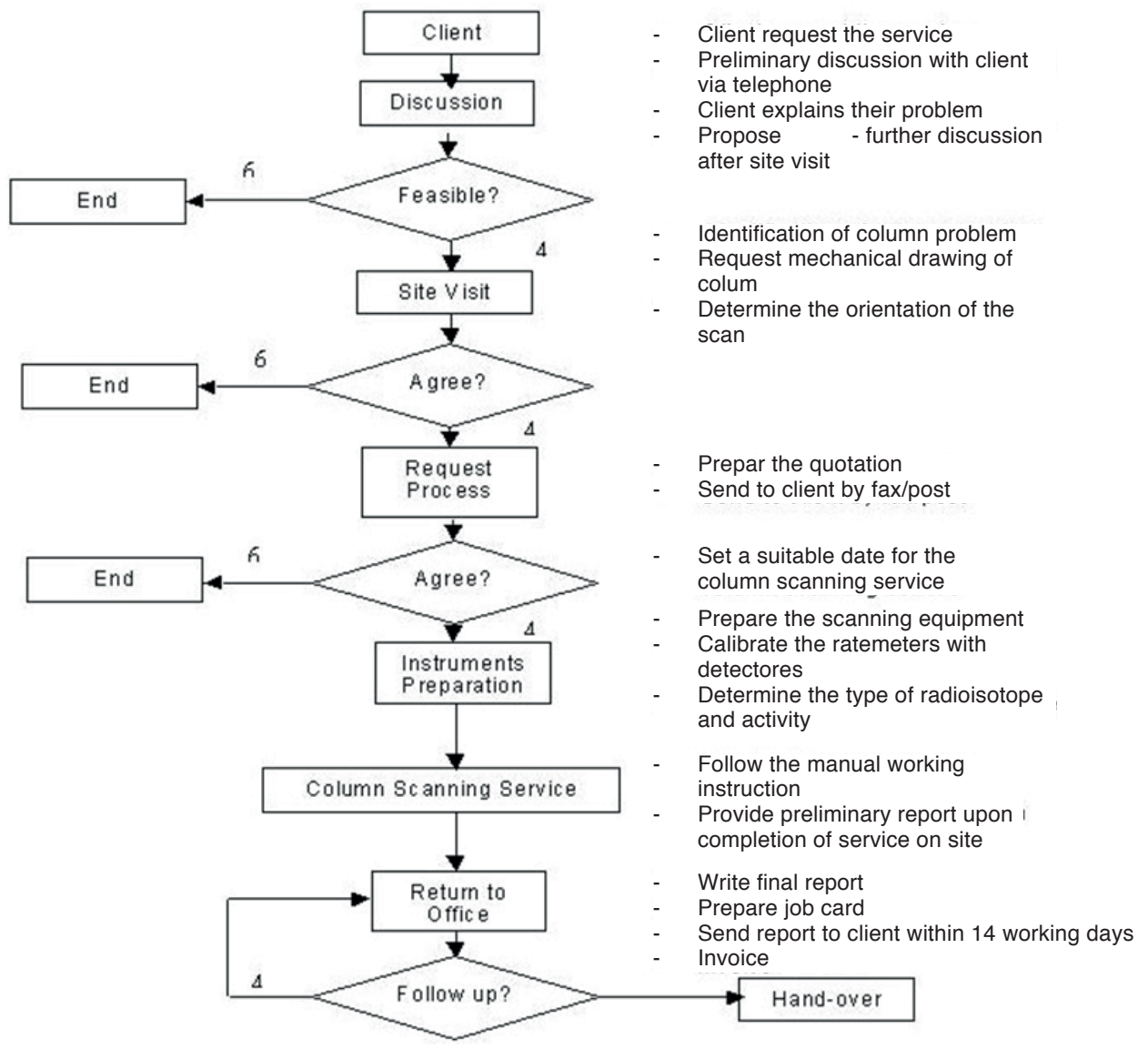

Fig. 9 Flow chart for column scanning services to satisfy customer requirements

All gamma-ray scanning services have to follow the policy and procedures as documented in the Quality Manual and Quality Procedures. Work instructions have to be written in layman's terms, easy to be understood by technician and supervisors. In this way, 
is also easier to integrate as part of the day-to-day operations. The system documentation is controlled, with each document containing a history of revisions and a distribution list and a signature page duly signed by relevant authorities.

Figure 9 shows a flow chart of the ways in which a Radioisotope Application Group could ensures gamma-ray scanning services provide to petroleum refineries, gas processing installations and chemical plants meet and satisfies customer requirements.

The quality policies of a radioisotope group should consider the following:

(i) Radioisotope group shall ensure that consistent quality will be guaranteed to all services rendered through Quality Management System (QMS) standard and continual improvement.

(ii) Radioisotope group shall ensure high priority to the safety aspects of its radiation workers, and a proper handling of radioactive sources, for the benefit, care and welfare of the public and the environment.

(iii) Radioisotope group shall strive in order to comply with legal requirements, either national or international standards.

The illustration in figure 8 represents a logical program structure; other formats may also be acceptable provided that the information meets the following criteria:

(i) Communicates specifications efficiently.

(ii) Demonstrates that all aspects which required control are indeed in control.

(iii) Permits regular assessment of program effectiveness by the licensee, the operator and the regulatory body.

Great efforts are also extended in the training of staff to familiarize themselves with the ISO 9001:2000 requirements. Several audits have to be performed to continuously review the critical process points, corrective action and outcomes. The final, so called Adequacy Audit, has to be conducted by a National or International body. The Compliance Audit for the purpose of registration comes after Adequacy Audit. The ISO 9001:2000 QMS certification is obtained if successfully passed the Audits. The first Surveillance Audit normally is carried out after one year period to certify or re-affirm the high quality of measurements performed by the company and instill confidence among its customers on the work that it carries out.

The sufficiently high quality services offered by a radioisotope application company according to the ISO 9001:2000 certification are as follows:

(i) A sufficiently accurate and reliable gamma-ray scanning system which is regularly calibrated and routinely checked.

(ii) Well-trained and experienced plant diagnostic service personnel.

(iii) On-time delivery of gamma-ray scanning services to the customers, including information about the results and information to the competent authorities.

(iv) Safe and sufficiently comprehensive storage of scanning results for long periods (i.e. 30 years).

(v) Effective internal quality systems and administration.

\section{Emphasis in certification of processes}

A radioisotope application group has to give more and more attention to the formal 
institutionalization of Quality Assurance/Quality Management practices for the regular functions, owing to, for example, the variety and complexity of the column scanning activities performed. The commitment of the radioisotope application group is expressed in a Policy Statement. This commitment is further expressed, which is stipulated by the involvement of management into a Management Review process. This encompasses the review and approval of documentation, internal audits and regular operational reviews involving management and site personnel. The process control of individual monitoring strategy differentiates issuance and evaluation of dosimeter. The overall service strategy is supported by various mechanisms to ensure proper assignment and the traceability of data. Scheduled audits are planned, as they serve as a guide to correct unnoticeable problem. They give great boost of confidence to attain a high degree of accuracy in the quality system.

Personnel in the column scanning team are trained on various disciplines in gammaray scanning activities. Basic training is carried out in a systematic manner to enable worker to adopt the techniques required to obtain quality column scanning services, as appropriate to the duties. It is provided to every new worker before starting work. The aim is to increase worker awareness and the understanding of specific job-related activities. Supervisor training is also essential to further integrate column scanning service procedures and work practices into the day-to-day staff activities. The ability to complete the tasks specified are recorded in a Practical Assessment Form and are kept at a Quality Assurance Section.

A formal Change of Management Process is implemented to handle changes to the system (i.e. procedures, work instructions, equipment etc.). This process puts emphasis on the facility critical systems. A formal process for identifying, correcting and documenting Non-Conformities is also implemented. Records, Assessment, Report and Communications are key milestones for the continued success of the Column Scanning Services. Regular meeting and reports on various technical and non-technical aspects involve a wide spectrum of staff, from laboratory assistant, technician, and supervisor to Section Managers. These meetings and reports have been instituted at an early stage of operation, and include frequent interactions with other divisions. Besides these meeting and reports, communication with the regulatory bodies and customer on items requiring follow-up actions are tracked in a proper record. Complaints from customers are recorded, analyzed and solved.

Customer satisfaction is recognized as one of the driving criteria for the sustainability of the process.

\section{Accreditation of radioisotope applications laboratories}

The ISO 9001:2000 Quality Management System has been successful in helping organizations to deliver quality products or services. However, it should be noted that the ISO 9000 series addressed only the management aspects of an organization. For laboratories providing calibration and testing services, there are loopholes that are not covered by this system. For this reason, the standard known as ISO 17025 (up to August 1990 it was known as ISO Guide 25) was introduced. In this standard, not only the management aspects of 
the organization were addressed, the technical aspects were also given a comprehensive coverage to ensure that an accredited laboratory would be able to deliver quality products or services.

However, there is question mark about the applicability of ISO 17025 for accreditation of radioisotope application laboratories. Many individual, especially those involved in providing services, for instance column scanning services at industrial process plants away from their premises are of the opinion that such a standard is only applicable for laboratories and not for testing, investigation or inspection activities performed at sites. However, this doubt was clarified by ISO 17025:

"The laboratory management system shall cover works carried out in the laboratory's permanent facilities, at sites away from its permanents facilities or is associated temporary or mobiles facilities".

For column scanning, both the radioisotope sealed source application laboratory which is used for conducting research and development works, and calibrating and testing of field equipments, among others, and the mobile laboratory for field investigation and services, will be accredited to ISO 17025 in order to win recognition from industrial community both locally and at the international level.

\section{REFERENCES}

J.B.Abdullah (2002). Gamma Scanning for Troubleshooting, Optimization and Predictive Maintenance of Distillation Columns in Petroleum Refineries and Chemical Plants, Proceedings of the International Nuclear Conference 2002, Kuala Lumpur, Malaysia

N.M.Ali (2003). Implementation of a Quality System Based on ISO 9001 Requirement in Malaysia National Dosimetry Services for Monitoring External Radiation Exposure, IAEA Conference on Radiation Protection, Rabat, Morocco.

M.L.Juri, S.Othman, T.Kadni, M.Wahid and D.Mohamad (1998). ISO 9000, En 4600 and Quality System Regulation (QSR, USFDA) : Fact and Fallacy, MINT's R\&D Seminar.

INTERNATIONAL STANDARD ISO 9001:2000. Quality Management Systems Requirements. http://www.sirim-qas.com.my

OIEA-ARCAL XLIII (2000). "Guía para Aplicaciones Industriales de Radiotrazadores y Fuentes Selladas de Radiación".

IAEA (1973). "Radiation Protection Procedures". Safety Series N³8, Viena.

International Atomic Energy Agency, (2002). Radioisotope Applications for Troubleshooting and Optimizing Industrial Processes, March.

J.S.Charlton (1986). Radioisotope Techniques for Problem-Solving in Industrial Process Plants. Leonard Hill.

A.E.Hills, J. Thereska (1999). Practical Guidebook for Radioisotope-Based Technology in Industry, IAEA/RCA RAS/8/078. 